\title{
Safety and efficacy of pegylated recombinant human granulocyte colony-stimulating factor during concurrent chemoradiotherapy for small-cell lung cancer: a retrospective, cohort-controlled trial
}

\section{Cunliang Wang}

Shandong First Medical University

Shouhui Zhu

Shandong Tumor Hospital

Chuanwang Miao

Shandong Tumor Hospital

\section{Yu Wang}

Shandong Second Provincial General Hospital

Jiazhen Chen

Shandong First Medical University

\section{Shuanghu Yuan}

Shandong Tumor Hospital

Xudong Hu ( $\nabla$ drhuxudong@163.com )

Shandong Tumor Hospital

\section{Research Article}

Keywords: Febrile neutropenia, Hematological toxicity, PEG-rhG-CSF, Radiotherapy, Survival time

Posted Date: January 10th, 2022

DOI: https://doi.org/10.21203/rs.3.rs-1205271/v1

License: (c) (1) This work is licensed under a Creative Commons Attribution 4.0 International License. Read Full License 


\section{Abstract \\ Objective}

To investigate pegylated recombinant human granulocyte colony-stimulating factor (PEG-rhG-CSF) safety and efficacy in preventing hematological toxicity during concurrent chemoradiotherapy (CCRT) for smallcell lung cancer (SCLC).

\section{Methods}

We retrospectively assessed 80 SCLC patients treated with CCRT from January 2013 to December 2018 who received PEG-rhG-CSF within 48 hours after the end of chemotherapy, defined as prophylactic use, as the experimental group. An additional 80 patients who were not treated with PEG-rhG-CSF were matched 1:1 by the propensity score matching method and served as the control group. The main observations were differences in hematological toxicity, neutrophil changes, febrile neutropenia (FN) incidence and adverse reactions. Progression-free survival (PFS) and overall survival (OS) were analyzed with regular assessment and follow-up.

\section{Results}

The leukocyte, neutrophil, erythrocyte, and platelet counts and hemoglobin level decreased after CCRT, but the experimental group had slightly higher leukocyte and neutrophil counts than the control group $(P<0.05)$. The incidences of grade III-IV leukopenia (18.75\% vs. $61.25 \%)$ and neutropenia $(23.75 \%$ vs. $67.5 \%)$ in the experimental group were significantly lower than those in the control group $(P<0.05)$. The absolute neutrophil count was $4.17 \pm 0.79$ on day 1 and peaked $(6.81 \pm 2.37)$ on day 10 in the experimental group; the value in the control group was $2.81 \pm 0.86$ on day 1 . It decreased significantly and reached the minimum $(0.91 \pm 0.53)$ on day $10(P<0.05)$. The experimental group had a lower $F N$ incidence than the control group $(P<0.05)$. There was also no significant acute esophagitis or pulmonary toxicity. The treatment had no significant effect on PFS (11.4 vs. 8.7, $\mathrm{P}=0.958)$ or $\mathrm{OS}(23.9$ vs. $17.3, \mathrm{P}=0.325)$ over an 18.6-month median follow-up time.

\section{Conclusion}

PEG-rhG-CSF has good efficacy and safety in preventing hematological toxicity in SCLC patients during CCRT and has no significant effects on PFS or OS.

\section{Introduction}

Small-cell lung cancer (SCLC) accounts for approximately $15 \%$ of all lung cancer cases. It is a poorly differentiated and highly malignant tumor with partial neuroendocrine characteristics, rapid growth, 
strong invasiveness, early metastasis and poor prognosis [1]. Limited-stage SCLC accounts for approximately $30 \%$ of all SCLC cases at the time of diagnosis [2]. Concurrent chemoradiotherapy (CCRT) can reduce tumor volume, suppress tumor blood supply and provide a synergistic sensitizing effect, and it is used to treat a wide range of solid tumors. CCRT is the standard treatment for patients with SCLC [3]. It can improve the efficacy of the treatment and produces a significant survival benefit. However, it can also increase the incidence of acute blood toxicity, which can reduce the dose of chemotherapy and delay treatment. Severe neutropenia can easily induce febrile neutropenia (FN), infectious toxic shock and death, which directly affects the clinical treatment and survival of patients.

A phase III randomized study by the Southwest Oncology Group in SCLC patients showed that the combination of CCRT with granulocyte-macrophage colony-stimulating factor (GM-CSF) significantly increased the incidence of toxic events, and the median survival duration was slightly shorter in GM-CSFtreated patients [4]. Therefore, based on this study, the National Comprehensive Cancer Network (NCCN) guidelines, do not recommend prophylactic use of granulocyte colony-stimulating factor (G-CSF) with CCRT; if granulocyte deficiency occurs during radiotherapy, it can be treated routinely with G-CSF. However, the Infectious Diseases Society of America (IDSA) guidelines [5] require that once FN is diagnosed, the treatment must be administered within $2 \mathrm{~h}$ because infection may progress very rapidly in patients with granulocyte deficiency. Therefore, the NCCN guidelines removed the non-recommendation for G-CSF prophylaxis during CCRT. In addition, modern precision radiotherapy techniques can significantly reduce toxic effects compared with previous two-dimensional techniques, and some data from relevant clinical trials show that G-CSF combined with radiotherapy and chemotherapy does not increase chest toxicity and has no effect on survival $[6,7]$.

Along with modern drug development and the development of newer iterations of G-CSF, the novel agent pegylated recombinant human granulocyte colony-stimulating factor (PEG-rhG-CSF), a long-acting, selfregulating rhG-CSF with reduced plasma clearance and prolonged half-life, warrants further evaluation to determine its value in clinical therapy. Some studies have shown that prophylactic administration of PEGrhG-CSF reduces the incidence, duration and severity of chemotherapy-associated neutropenia in patients with cervical cancer, breast cancer and other tumors [8-12]. Studies employing PEG-rhG-CSF in SCLC are sparse. To explore whether PEG-rhG-CSF can be applied during CCRT for SCLC, a retrospective, cohortcontrolled trial was carried out to evaluate the efficacy and safety in preventing hematological toxicity of PEG-rhG-CSF for SCLC during CCRT.

\section{Materials And Methods}

\subsection{Study design and inclusion criteria}

This was a retrospective cohort study of all patients diagnosed with SCLC and treated with CCRT in the radiotherapy department of Shandong Cancer Hospital from 2013 to 2018. Eighty patients met the inclusion criteria and received PEG-rhG-CSF within $48 \mathrm{~h}$ after the end of each cycle of chemotherapy during CCRT. This was defined as prophylactic use, and these patients served as the experimental group. 
After controlling for age, sex, smoking history and other factors, a further 80 patients were identified by the 1:1 propensity score matching method. In addition to meeting the basic inclusion criteria, they did not receive PEG-rhG-CSF treatment within $48 \mathrm{~h}$ after the end of chemotherapy during CCRT and only received therapeutic rhG-CSF when bone marrow suppression occurred; therefore, these patients served as the control group.

The inclusion criteria were as follows: (1) patients with pathologically confirmed SCLC who had been treated with 2 cycles of etoposide plus cisplatin (EP) regimen chemotherapy; (2) patients treated with CCRT at the end of 2 cycles of chemotherapy and 2 cycles of concurrent chemotherapy during radiotherapy; (3) patients with an absolute neutrophil count (ANC) $<1 \times 10^{9} / \mathrm{L}$ after the previous cycle of chemotherapy; (4) patients with a white blood cell (WBC) count $>4 \times 10^{9} / \mathrm{L}$ and ANC $>2 \times 10^{9} / \mathrm{L}$ before this CCRT; (5) patients without liver or kidney dysfunction, hematologic disorders or other malignant tumors, aged $\geq 18$ years but $\leq 70$ years, and with Karnofsky performance status (KPS) of $\geq 80$ points; (6) patients who had not received prior radiotherapy; and (7) patients with complete and traceable imaging data.

\subsection{Treatment}

Patients in both groups were treated with two cycles of chemotherapy as follows: etoposide $100 \mathrm{mg} / \mathrm{m}^{2}$ ivdrip on days 1 to 5 and cisplatin $40 \mathrm{mg} / \mathrm{m}^{2}$ ivdrip on days 1 to 3 . The EP regimen was given every 3 weeks as a chemotherapy cycle. Radiotherapy was given at the start of chemotherapy with the same total dose of $60 \mathrm{~Gy} / 30$ times, 5 times/week.

The experimental group was injected subcutaneously with $6 \mathrm{mg}$ PEG-rhG-CSF within $48 \mathrm{~h}$ after the end of each cycle of chemotherapy during CCRT, while in the control group, PEG-rhG-CSF was not administered prophylactically. If the patients had $\mathrm{WBC}<3 \times 10^{9} / \mathrm{L}$ or $\mathrm{ANC}<1 \times 10^{9} / \mathrm{L}$ during the treatment, $5 \mu \mathrm{g} / \mathrm{kg} / \mathrm{d}$ rhGCSF was injected subcutaneously, and both chemotherapy and radiotherapy were stopped until ANC $\geq 2 \times 10^{9} / L$ was achieved.

\subsection{Efficacy assessment}

(1) Complete blood cell counts, mainly assessing the WBC count, ANC, red blood cell (RBC) count, hemoglobin (HB) level and platelet (PLT) count, were performed every week during CCRT, and the values were compared. (2) The ANCs on days 1, 5, 10, 15 and 20 of the first cycle of concurrent chemotherapy were recorded for comparison between the two groups $[13,14]$. (3) Patients with interrupted radiotherapy, radioactive reactions, incidences of FN and adverse reactions were compared between the two groups. (4) Progression-free survival (PFS) was defined as the interval from the first day of CCRT to the first sign of disease progression or death. Overall survival (OS) was determined as the interval from the first day of CCRT to death from any cause. All imaging examinations were re-evaluated to determine progression and record the PFS. OS was analyzed by calling or texting the patients and reviewing patient information. Statistical significance was indicated by $\mathrm{P}<0.05$.

\subsection{Statistical analysis}


Data analysis was performed using SPSS 21.0 software. For continuous variables, the normality of the two groups was tested by the Shapiro-Wilk (S-W) method. If the data obeyed a normal distribution, they were analyzed by two-independent-sample t-tests and the data were expressed as the mean \pm standard deviation. If the data did not conform to a normal distribution, they were analyzed by non-parametric Friedman tests. Classified data were analyzed by the $\chi^{2}$ test. $P<0.05$ indicated that the difference was statistically significant. Categorical variables were expressed as composition ratios.

\section{Results}

\subsection{Baseline characteristics of the patients}

A total of 160 patients were enrolled in this trial. They were divided into two groups, namely, the PEG-rhGCSF experimental group and the control group, with 80 participants per group. Table 1 summarizes the baseline characteristics of the patients. The average age of the experimental group was 59.70 years, and that of the control group was 59.33 years. Both groups were predominantly male, and more than half of the patients had a history of smoking. According to the Veterans' Administration Lung Study Group (VALSG) staging system [15], there were 7 patients with extensive stage disease and 73 patients with limited stage disease in the experimental group. The control group included 8 patients with extensive stage disease and 72 patients with limited stage disease. There was no significant difference between the two groups of patients in various baseline characteristics $(P>0.05)$.

Table 1

Patient characteristics at baseline.

\begin{tabular}{|lllll|}
\hline Characteristic & Experimental group $(\mathbf{N}=\mathbf{8 0})$ & $\begin{array}{l}\text { Control group } \\
(\mathbf{N}=80)\end{array}$ & Statistics & P \\
\hline Age (years) & $59.70 \pm 8.12$ & $59.33 \pm 9.66$ & $\mathrm{t}=0.275$ & 0.784 \\
\hline Sex & 64 & & $\chi 2=0.040$ & 0.841 \\
\hline Male & 16 & 65 & & \\
\hline Female & 50 & 15 & & \\
\hline Smoking history & & & & \\
\hline Smoker & 30 & 46 & & \\
\hline Never smoker & & 34 & & \\
\hline Stage & 7 & & & \\
\hline Extensive stage & 7 & 8 & & \\
\hline Limited stage & 73 & 72 & & \\
\hline 3.2. Blod count differences between the two groups
\end{tabular}


There were no significant differences between the two groups in WBC count, ANC, RBC count, PLT count or HB level before CCRT $(P>0.05)$. However, after CCRT, the counts decreased to varying degrees, and the WBC count and ANC of the experimental group were slightly higher than those of the control group; the difference was significant $(P<0.05)$. The RBC count, PLT count and HB level showed no significant changes after CCRT, as shown in Figures 1A-E (Supplementary Table 1).

CCRT: concurrent chemoradiotherapy; WBC: white blood cell; ANC: absolute neutrophil count; RBC: red blood cell; PLT: platelet; HB: hemoglobin.

* Factors with statistical significance: ns: no significance; $*: p<0.05 ; * *: p<0.01 ; * * *: p<0.001$.

\subsection{Changes in ANC}

Figure 2 show the changes in ANC on days 1, 5, 10, 15, and 20 (D1, D5, D10, D15, and D20, respectively) in both groups of patients. The ANC was $4.17 \pm 0.79$ in the experimental group and $2.81 \pm 0.86$ in the control group on D1. It reached a peak of $6.81 \pm 2.37$ on D10 after the administration of PEG-rhG-CSF in the experimental group, while it decreased significantly in the control group, reaching a minimum of 0.91 \pm 0.53 ; the difference was statistically significant $(P<0.05$; Supplementary Table 2$)$. During CCRT, although rhG-CSF was given when grade III or higher neutropenia occurred in the control group, the ANC was still lower than that in the experimental group. During the treatment, a total of 15 people in the experimental group had delayed treatment because of granulocytopenia, compared to 29 people in the control group. The difference was significant $(P=0.013)$.

\subsection{Hematological toxicity}

According to the World Health Organization's criteria for acute and subacute toxic reactions, postchemotherapy myelosuppression is classified as grade 0-IV [16]. The hematological toxicity data are shown in Figures 3A-D (Supplementary Table 3). The total incidences of leukopenia (93.75\% vs. 100.0\%) and neutropenia ( $81.25 \%$ vs. $93.75 \%)$ in the experimental group were lower than those in the control group $(P<0.05)$, and the incidences of grade III-IV leukopenia ( $18.75 \%$ vs. $61.25 \%)$ and neutropenia $(23.75 \%$ vs. $67.5 \%)$ in the experimental group were also significantly lower than those in the control group $(\mathrm{P}<0.05)$. However, there were no significant differences in the incidence of grade III-IV thrombocytopenia ( $8.75 \%$ vs. $22.5 \%)$ or anemia (10.0\% vs. $17.5 \%)$ between the two groups $(P>0.05)$.

* Factors with statistical significance: ns: no significance; $*$ : $p<0.05 ; * *: p<0.01 ; * * *: p<0.001$.

\subsection{Adverse reactions}

The main side effects of treatment in all patients were radiation pneumonia and esophagitis. Based on the Radiation Therapy Oncology Group (RTOG) grading scale for acute radiation injury [17], 2 patients in the experimental group and 1 patient in the control group developed 1 st-degree radiation pneumonia. The number of patients with 1st -, 2nd - and 3rd-degree esophagitis was 14, 11 and 2 in the experimental group and 19, 5 and 0 in the control group, respectively. None of the differences were significant $(P>0.05)$, 
as shown in Figure 4A. FN occurred in 2 patients (2.5\%) in the experimental group and occurred in 13 patients (16.25\%) in the control group, and the incidence was significantly lower in the experimental group than in the control group $(P=0.003)$. Other adverse reactions of patients in both groups were mainly bone pain, palpitation and weakness, and the differences were not statistically significant $(P>0.05)$. Patients with bone pain had relatively mild symptoms that dissipated without intervention, and other adverse reactions were relieved after discontinuation of the drug and administration of symptomatic treatment, as shown in Figure 4B (Supplementary Table 4).

RP: Radiation pneumonia, RE: Radiation esophagitis, FN: Febrile neutropenia

* Factors with statistical significance: ns: no significance; $*$ : $p<0.05 ; * *: p<0.01 ; * * *: p<0.001$.

\subsection{Survival}

A total of 13 people were lost to follow-up, namely, 9 in the experimental group and 4 in the control group. The follow-up period was 0.7-96.1 months as of May 1, 2021, with a median follow-up period of 18.6 months. Prophylactic application of PEG-rhG-CSF during CCRT had no significant effects on PFS (11.4 vs. $8.7 ; P=0.756)$ or $O S(23.9$ vs. $17.3 ; P=0.325)$. The differences were not statistically significant, as shown in Figure 5A, 5B.

\section{Discussion}

The most common side effect during CCRT is blood cell toxicity. In severe cases, it can induce FN, and the immune decline triggers infections and can even be life-threatening $[18,19]$. At present, rhG-CSF and PEGrhG-CSF are routinely used in antitumor adjuvant therapy, and PEG-rhG-CSF has shown more strengths. A large number of domestic and international studies have confirmed that PEG-rhG-CSF has similar efficacy to rhG-CSF but has superior safety in the prophylactic treatment of chemotherapy-induced non-myeloidderived neutropenia [20-24]. RhG-CSF requires daily administration because of its short half-life [25-27]. However, PEG-rhG-CSF is a long-acting, self-regulating rhG-CSF that has reduced plasma clearance and prolonged half-life compared to rhG-CSF, with only one dose required per chemotherapy cycle $[26,28,29]$. Therefore, it is more favorable for clinical treatment.

Research has shown that concurrent use of chemotherapy and administration of hematological growth factors may enhance hematological toxicity [30]. The initial evidence came from a multicenter prospective trial by the Southwest Oncology Group [4]. The results show that patients administered GMCSF had higher WBC counts and ANCs, but there was no significant difference in the incidence of grade IV leukopenia or neutropenia. However, there was a statistically significant increase in the incidences of thrombocytopenia and anemia in the GM-CSF arm. The rates of infection and toxicity-related death were also higher in GM-CSF patients.

Early studies focused on GM-CSF, which does not act specifically on granulocyte progenitors, and it is no longer a routine treatment used to raise leukocyte counts. Therefore, it is necessary to re-evaluate the safety of granulocyte-stimulating factors. Some studies have shown that the use of granulocyte- 
stimulating factors is justified [31]. In addition, a randomized controlled trial and meta-analysis indicated that the incidences of neutrophil deficiency and FN were significantly improved with the use of longacting G-CSF [32]. Goodman Lindsey Martin et al analyzed the prophylactic use of PEG-rhG-CSF in 180 patients with NSCLC receiving new chemotherapy regimens and showed a significant reduction in the FN risk [33]. In the past, G-CSF was given to patients deemed to be at high risk of developing hematological toxicity. However, the results of the available studies contradict previous perceptions. Patients treated with G-CSF have significantly lower hematological toxicity and may have better treatment efficacy. The results of our trial further confirm this.

A phase II clinical trial of prophylactic application of G-CSF in 38 patients with limited-stage SCLC treated with CCRT did not show an increased risk of acute or advanced pulmonary toxicity or grade III-IV acute esophagitis, nor did it result in treatment-related mortality in patients. There was no significant difference in the incidence of neutropenia, but the incidence of grade III/IV adverse events was slightly lower than that in the non-preventive use group. However, prophylactic application of G-CSF increased the risk of thrombocytopenia [7]. This result is similar to that of the trial by the Southwest Oncology Group. Our study suggests that the PLT counts is decreased with PEG-rhG-CSF administration, and the decrease after therapy was still lower than that in patients not treated with PEG-rhG-CSF. The grade III-IV

thrombocytopenia incidence was lower than in patients who did not use PEG-rhG-CSF. Although there was no difference in the PLT count, there were also no bleeding events due to thrombocytopenia during the treatment. Therefore, the reduction in the PLT count may be a manifestation of myelosuppression and not closely related to PEG-rhG-CSF administration. CONVERT is a phase III randomized controlled trial involving 547 patients with SCLC treated with CCRT [6]. Of the patients, 33\% received at least one cycle of prophylactic G-CSF, and $41 \%$ received therapeutic G-CSF. The application of G-CSF during CCRT did not increase the risk of acute esophagitis or pulmonary toxicity and facilitated treatment completion. In contrast, there was a predominance of grade I and II esophagitis, with very few patients having symptoms of pneumonia and no cases of severe pulmonary toxicity or esophagitis in our study. The patients received appropriate therapy to alleviate these reactions during treatment and did not feel otherwise unwell. Therefore, these research findings indicate that the administration of PEG-rhG-CSF is reliable in terms of safety and efficacy. It is feasible to prophylactically administer granulocyte-stimulating factor.

Previous studies by the Southwest Oncology Group have shown that the median survival duration is slightly shorter in G-CSF-treated patients than in patients treated without G-CSF. However, G.H. Lyman et al conducted a systematic review and meta-analysis of the effect of G-CSF on the chemotherapy dose and survival of oncology patients [34]. Patients treated with G-CSF had reduced all-cause mortality. Liu Fang performed a retrospective study of 33 patients with non-small-cell lung cancer (NSCLC) who had previously developed FN, and 29 patients received PEG-rhG-CSF prophylactically at the start of the next cycle of chemotherapy [35]. The median PFS and OS times for patients treated with and without PEG-rhGCSF prophylactically were 177 and 163 days ( $P F S ; P=0.20$ ), 628 days and 274 days (OS; $P=0.13$ ), respectively, which are similar to the results of our study. These results further illustrate the reliability of PEG-rhG-CSF. Prophylactic use of PEG-rhG-CSF may reduce the probability of radiotherapy-related 
adverse events and make oncology treatment safe and effective. More importantly, it had no significant impact on survival time.

Our study focused on hematological toxicity with PEG-rhG-CSF administration during CCRT. The results showed that prophylactic application of PEG-rhG-CSF significantly reduced the incidences of leukopenia and neutropenia, especially the incidences of grade III-IV leukopenia and neutropenia. More importantly, there were no significant differences in the incidences of grade III-IV thrombocytopenia and anemia between the groups, and there were no cases of blood transfusion, death or other serious adverse events. Furthermore, on the 10th day after chemotherapy, the experimental group achieved the peak ANC and thereafter maintained normal neutrophil levels, while the control group showed varying degrees of reduction despite regular administration of rhG-CSF. Importantly, the number of patients with radiotherapy interruptions was significantly lower in the experimental group than in the control group. In addition, the incidence of FN was also significantly decreased, and the incidences of all other adverse reactions were within the control ranges. There was also no significant acute esophagitis or pulmonary toxicity. Furthermore, no toxicity-related deaths were observed, and the results showed no effects on PFS or OS. These findings show that PEG-rhG-CSF has good reliability and compliance.

\section{Conclusion}

The prophylactic administration of PEG-rhG-CSF can significantly reduce the incidences of leukopenia and neutropenia, especially the incidence of grade 3 or higher hemocytopenia events, and can significantly reduce the incidence of FN and the frequency of treatment interruptions. More importantly, prophylactic administration of PEG-rhG-CSF did not lead to a significant effect on survival time. This result indicates that PEG-rhG-CSF has good efficacy and safety in preventing hematological toxicity in SCLC patients during CCRT. The clinical analysis of this study was biased due to the case selection, small sample size, and small number of patients who missed visits; therefore, further validation with a larger sample or related prospective studies are needed. However, the results of this article are also informative for clinical guidance.

\section{Abbreviations}

PEG-rhG-CSF, Pegylated recombinant human granulocyte colony-stimulating factor; CCRT, Concurrent chemoradiotherapy; SCLC, Small-cell lung cancer; FN, Febrile neutropenia; PFS, Progression-free survival; OS, Overall survival; GM-CSF, Granulocyte-macrophage colony-stimulating factor; NCCN, National Comprehensive Cancer Network; G-CSF, Granulocyte colony-stimulating factor; IDSA, Infectious Diseases Society of America; ANC, Absolute neutrophil count; KPS, Karnofsky performance status; WBC, White blood cell; RBC, Red blood cell; PLT, Platelet; HB, Hemoglobin; RP: Radiation pneumonia, RE: Radiation esophagitis.

\section{Declarations}




\section{Ethics approval and consent to participate}

The need for informed consent was waived because of the retrospective nature of the study.

\section{Consent for publication}

Not applicable.

\section{Availability of data and materials}

The datasets generated during and/or analyzed during the current study are not publicly available. But the data are available from the corresponding author upon reasonable request.

\section{Competing interests}

All authors have no conflicts of interest to declare.

\section{Fundings}

This study was supported by the the CSCO-Qilu Oncology Research Fund (grant number Y-Q201801-054); the Projects of the Medical and Health Technology Development Program in Shandong Province (grant number 202009030676); and the Wu Jieping Medical Foundation (grant number 320.6750.2021-02-42).

\section{Author contributions}

Xudong Hu: conceptualization, study design, project administration, funding acquisition. Shouhui Zhu and Chuanwang Miao: supervision, formal analysis. Yu Wang: software, validation. Jiazhen Chen: data curation. Shuanghu Yuan: study design, methodology. Cunliang Wang: writing-original draft. Xudong Hu and Chuanwang Miao: writing-review \& editing. All authors: final approval of the manuscript.

\section{Acknowledgments}

We would like to sincerely thank all the patients who were included in this study and their families and all our colleagues for their contributions to this trial. 


\section{Author details}

${ }^{1}$ Shandong First Medical University, Jinan 250000, Shangdong

${ }^{2}$ Department of Radiation Oncology, Shandong Cancer Hospital and Institute, Shandong First Medical University and Shandong Academy of Medical Sciences, Jinan 250117, Shandong

${ }^{3}$ Department of Radiation Oncology, Shandong Second Provincial General Hospital Jinan 250022, Shandong

\section{Supplementary Information}

Additional file 1: S1 Table. Blood counts differences. These data did not obey a normal distribution according to the Shapiro-Wilk test $(P<0.05)$, so nonparametric tests on two independent samples were used for comparison. S2 Table. Changes in ANC (x109/L). The data, which did not obey the normal distribution according to the Shapiro-Wilk test (Pख0.05), so a nonparametric (Friedman) test was used. S3 Table. Hematological toxicity incidence in the two groups (\% patients). S4 Table. Incidence of FN and other adverse reactions in the two groups. FN: febrile neutropenia

\section{References}

1. Zhang X. Interpretation of CSCO Guidelines for the Diagnosis and Treatment of Small Cell Lung Cancer in 2020. Journal of Clinical Internal Medicine 2020,37(11):820-822. https://doi.org/10.3969/j.issn.1001-9057.2020.11.021.

2. R. Govindan, N. Page, D. Morgensztern, W. Read, R. Tierney, A. Vlahiotis, E.L. Spitznagel, J. Piccirillo, Changing epidemiology of small-cell lung cancer in the United States over the last 30 years: analysis of the surveillance, epidemiologic, and end results database, J Clin Oncol 24(28) (2006) 453944.https://doi.org/10.1200/JC0.2005.04.4859

3. Chen M, Wang LH. Clinical Guidelines for Radiotherapy of Small Cell Lung Cancer in China (2020 Edition). Chinese Journal of Radiation Oncology 2020,29(08):608-614. https://doi.org/10.3760/cma.j.cn113030-20200528-00285.

4. P.A. Bunn, J. Crowley, K. Kelly, M.B. Hazuka, K. Beasley, C. Upchurch, R. Livingston, G.R. Weiss, W.J. Hicks, D.R. Gandara, Chemoradiotherapy with or without granulocyte-macrophage colony-stimulating factor in the treatment of limited-stage small-cell lung cancer: a prospective phase III randomized study of the Southwest Oncology Group, Journal of clinical oncology: official journal of the American Society of Clinical Oncology 13(7) (1995) 1632-1641

5. C.M.A. Chinese Society of Hematology, H.B. Chinese Medical Doctor Association, [Chinese guidelines for the clinical application of antibacterial drugs for agranulocytosis with fever (2020)], Zhonghua 
Xue Ye Xue Za Zhi 41(12) (2020) 969-978.https://doi.org/10.3760/cma.j.issn.02532727.2020.12.001

6. F. Gomes, C. Faivre-Finn, H. Mistry, A. Bezjak, N. Pourel, P. Fournel, J. Van Meerbeeck, F. Blackhall, Safety of G-CSF with concurrent chemo-radiotherapy in limited-stage small cell lung cancer Secondary analysis of the randomised phase 3 CONVERT trial, Lung Cancer 153 (2021) 165170.https://doi.org/10.1016/j.lungcan.2021.01.025

7. H. Sheikh, R. Colaco, P. Lorigan, F. Blackhall, R. Califano, L. Ashcroft, P. Taylor, N. Thatcher, C. FaivreFinn, Use of G-CSF during concurrent chemotherapy and thoracic radiotherapy in patients with limited-stage small-cell lung cancer safety data from a phase II trial, Lung cancer (Amsterdam, Netherlands) 74(1) (2011) 75-79.https://doi.org/10.1016/j.lungcan.2011.01.020

8. Liang XM, Huang WB. Clinical study of pegylated recombinant human granulocyte colonystimulating factor in the treatment of agranulocytosis after neoadjuvant chemotherapy for breast cancer. China Practical Medicine 2021,16(15):24-27. https://doi.org/10.14163/j.cnki.115547/r.2021.15.008.

9. Lin QF, Chen J, Zhang YH, et al. Clinical study on the prevention of post-chemotherapy leukopenia in lung cancer with pegylated recombinant human granulocyte colony-stimulating factor. Chinese Clinical Doctor 2020,48(12):1423-1426. https://doi.org/10.3969/j.issn.2095-8552.2020.12.012

10. Ma YM, Zhang MC, Zhang XH, et al. Prevention value of PEG-rhG-CSF for cervical cancer with concurrent Chemoradiotherapy. Medical Journal of Wuhan University 2020,41(01):44-47. https://doi.org/10.14188/j.1671-8852.2018.1185.

11. Mei ZJ, Jiang YF, Qiu H, et al. Primary prophylaxis treatment with PEG \rhG凶CSF for cervical cancer patients improves the neutropenia and life quality of patients: A cohort study. Medical Journal of Wuhan University 2020,41(02):240-244. https://doi.org/10.14188/j.1671-8852.2019.0264.

12. Ren JX, Zhao L, Tian XG, et al. Safety and efficacy of polyethylene glycol recombinant human granulocyte colony-stimulating factor in concurrent chemoradiotherapy for cervical cancer. Chinese Journal of Hospital Pharmacy 2021,41(06):632-636. https://doi.org/10.13286/j.10015213.2021.06.14

13. Xu SZ, Chen SR. Analysis of the efficacy of recombinant human granulocyte colony-stimulating factor in the prevention of myelosuppression after chemotherapy for breast cancer. Jilin Medical Journal 2019,40(03):550-551.

14. Zhang JW. Effect of PEGylated recombinant human granulocyte colony-stimulating factor on prevention of neutropenia after chemotherapy for small cell lung cancer. (Master's thesis, Tianjin Medical University).

15. M. Zelen, Keynote address on biostatistics and data retrieval, Cancer Chemother Rep 3 4(2) (1973) $31-42$

16. Wu YL, Cui XL, Yuan YH, Zhao ZG. Prevention and Treatment of Bone Marrow Suppression Caused by Antineoplastic Agents. Drug Evaluation 2010,7(14):30-36. 
17. J.D. Cox, J. Stetz, T.F. Pajak, Toxicity criteria of the Radiation Therapy Oncology Group (RTOG) and the European Organization for Research and Treatment of Cancer (EORTC), Int J Radiat Oncol Biol Phys 31(5) (1995) 1341-6.https://doi.org/10.1016/0360-3016(95)00060-C

18. Fan K, Dai LM, Wu ZF, et al. Advances in chemotherapy-induced myelosuppression. China J Tradit Chin Med Pharm 2017,32(01):210-214.

19. Xia XY, Fang WZ, Song HT. Effect of recombinant human granulocyte colony-stimulating factor on myelosuppression in tumor patients after the chemotherapy. Central South Pharm 2012,10(03):229232. https://doi.org/10.3969/j.issn.1672-2981.2012.03.020.

20. A. Mouri, K. Kaira, A. Shiono, O. Yamaguchi, Y. Murayama, K. Kobayashi, H. Kagamu, Clinical significance of primary prophylactic pegylated-granulocyte-colony stimulating factor after the administration of ramucirumab plus docetaxel in patients with previously treated non-small cell lung cancer, Thorac Cancer 10(4) (2019) 1005-1008.https://doi.org/10.1111/1759-7714.13022

21. F.P. Wu, J. Wang, H. Wang, N. Li, Y. Guo, Y.J. Cheng, Q. Liu, X.R. Yang, Clinical observation of the therapeutic effects of pegylated recombinant human granulocyte colony-stimulating factor in patients with concurrent chemoradiotherapy-induced grade IV neutropenia, Exp Ther Med 9(3) (2015) 761-765.https://doi.org/10.3892/etm.2014.2160

22. B. Yan, W. Zhang, F. Lu, Z.-L. Chen, B.-H. Han, L.-Y. Jiang, Safety of polyethylene glycol recombinant human granulocyte colony-stimulating factor in treating non-small cell lung cancer patients at I b stage, Asian Pac J Trop Med 6(11) (2013) 912-915.https://doi.org/10.1016/S1995-7645(13)601637

23. C. Zhou, Y. Huang, D. Wang, C. An, F. Zhou, Y. Li, G. Chen, C. Wu, J. He, G. Wu, X. Song, J. Gao, W. Liu, B. Li, J. Shi, C. Huang, J. Yu, J. Feng, H. Yue, M. Shi, J. Xia, A Randomized Multicenter Phase III Study of Single Administration of Mecapegfilgrastim (HHPG-19K), a Pegfilgrastim Biosimilar, for Prophylaxis of Chemotherapy-Induced Neutropenia in Patients With Advanced Non-Small-Cell Lung Cancer (NSCLC), Clin Lung Cancer 17(2) (2016) 119-127.https://doi.org/10.1016/j.cllc.2015.12.002

24. Zhang XQ, Zhu SH, Liu N, Wang Y, et al. Clinical observation of PEG-rhG-CSF in preventing neutropenia in 80 patients with small cell lung cancer treated with concurrent chemoradiotherapy. Journal of Shandong University (Health Sciences) 2020,58(12):43-46+59. https://doi.org/10.6040/j.issn.1671-7554.0.2020.09255

25. B.V. Fortner, A.C. Houts, L.S. Schwartzberg, A prospective investigation of chemotherapy-induced neutropenia and quality of life, J Support Oncol 4(9) (2006) 472-8

26. H. Tan, K. Tomic, D. Hurley, G. Daniel, R. Barron, J. Malin, Comparative effectiveness of colonystimulating factors for febrile neutropenia: a retrospective study, Curr Med Res Opin 27(1) (2011) 7986.https://doi.org/10.1185/03007995.2010.536527

27. S. Wetten, X. Li, J. Haas, G. Worth, C. Jacob, S. Braun, S. Tzivelekis, Comparative Effectiveness Of Granulocyte Colony-Stimulating Factors (G-Csf) For Reducing Incidence Of Febrile Neutropenia (Fn) -Related Hospitalization: A Retrospective Cohort Study Using German Claims Data, Value in Health 18(7) (2015).https://doi.org/10.1016/j.jval.2015.09.1042

Page $13 / 18$ 
28. J.L. Gabrilove, An analysis of current neutropenia therapies, including pegfilgrastim, Clin Cornerstone 8 Suppl 5 (2006) S19-28.https://doi.org/10.1016/s1098-3597(06)80055-4

29. Y. Zhai, Y. Zhao, J. Lei, Z. Su, G. Ma, Enhanced circulation half-life of site-specific PEGylated rhG-CSF: optimization of PEG molecular weight, J Biotechnol 142(3-4) (2009) 25966.https://doi.org/10.1016/j.jbiotec.2009.05.012

30. N.J. Meropol, L.L. Miller, E.L. Korn, L.E. Braitman, M.L. MacDermott, L.M. Schuchter, Severe myelosuppression resulting from concurrent administration of granulocyte colony-stimulating factor and cytotoxic chemotherapy, J Natl Cancer Inst 84(15) (1992) 1201-1203

31. F. Grossi, M. Tiseo, Granulocyte growth factors in the treatment of non-small cell lung cancer (NSCLC), Crit Rev Oncol Hematol 58(3) (2006) 22130.https://doi.org/10.1016/j.critrevonc.2005.09.002

32. L. Wang, O. Baser, L. Kutikova, J.H. Page, R. Barron, The impact of primary prophylaxis with granulocyte colony-stimulating factors on febrile neutropenia during chemotherapy: a systematic review and meta-analysis of randomized controlled trials, Support Care Cancer 23(11) (2015) 313140.https://doi.org/10.1007/s00520-015-2686-9

33. L.M. Goodman, M.B. Moeller, A.-G. Azzouqa, A.E. Guthrie, C.K. Dalby, M.A. Earl, C. Cheng, N.A. Pennell, M. Shapiro, V. Velcheti, J.P. Stevenson, Reduction of Inappropriate Prophylactic Pegylated Granulocyte Colony-Stimulating Factor Use for Patients With Non-Small-Cell Lung Cancer Who Receive Chemotherapy: An ASCO Quality Training Program Project of the Cleveland Clinic Taussig Cancer Institute, J Oncol Pract 12(1) (2016) e101-e107.https://doi.org/10.1200/JOP.2015.006502

34. G.H. Lyman, D.C. Dale, E. Culakova, M.S. Poniewierski, D.A. Wolff, N.M. Kuderer, M. Huang, J. Crawford, The impact of the granulocyte colony-stimulating factor on chemotherapy dose intensity and cancer survival: a systematic review and meta-analysis of randomized controlled trials, Ann Oncol 24(10) (2013) 2475-2484.https://doi.org/10.1093/annonc/mdt226

35. F. Liu, Y. Du, B. Cai, M. Yan, W. Yang, Q. Wang, A clinical study of polyethylene glycol recombinant human granulocyte colony-stimulating factor prevention neutropenia syndrome in patients with esophageal carcinoma and lung cancer after concurrent chemoradiotherapy, Journal of cancer research and therapeutics 13(5) (2017) 790-795.https://doi.org/10.4103/jcrt.JCRT_320_17

\section{Figures}


A

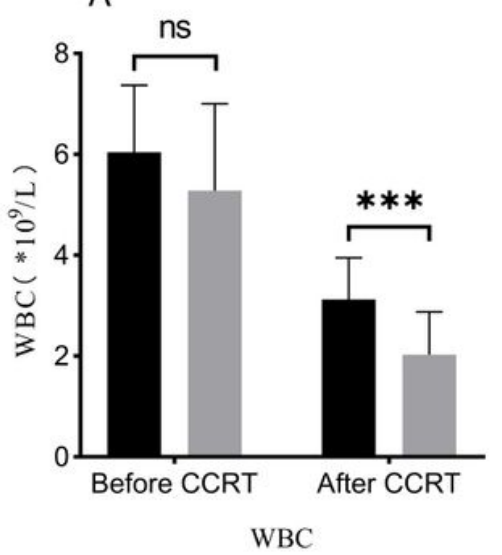

D

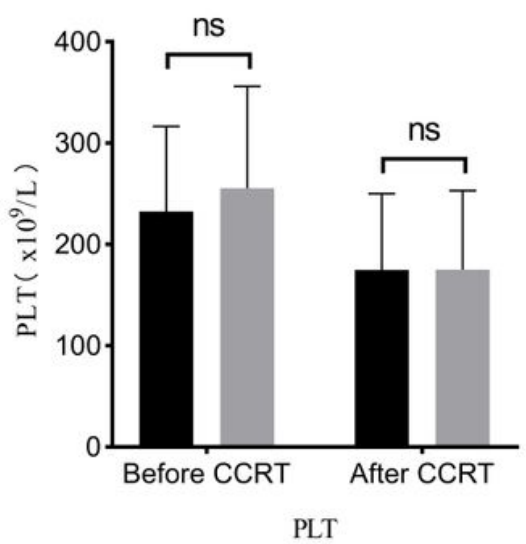

B

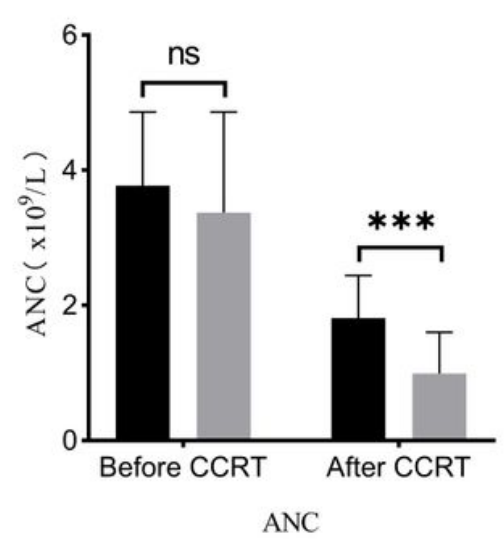

E

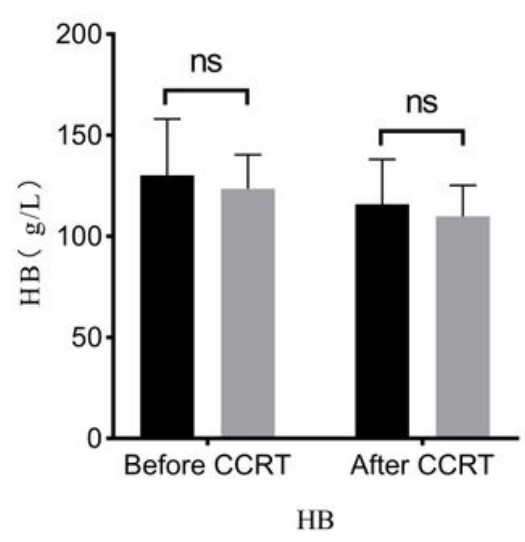

C

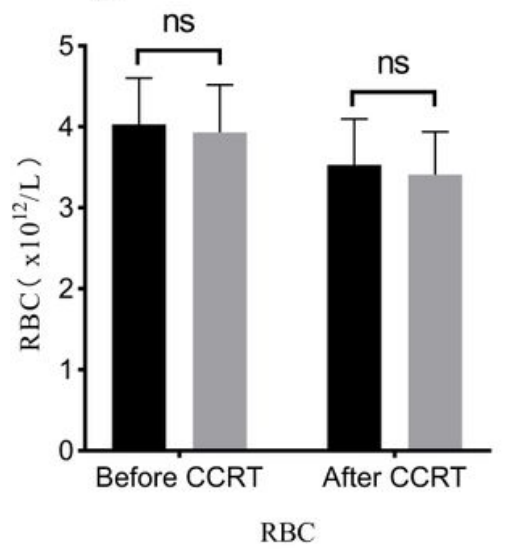

Experimental group

Control group

Figure 1

Blood count differences between the two groups. 


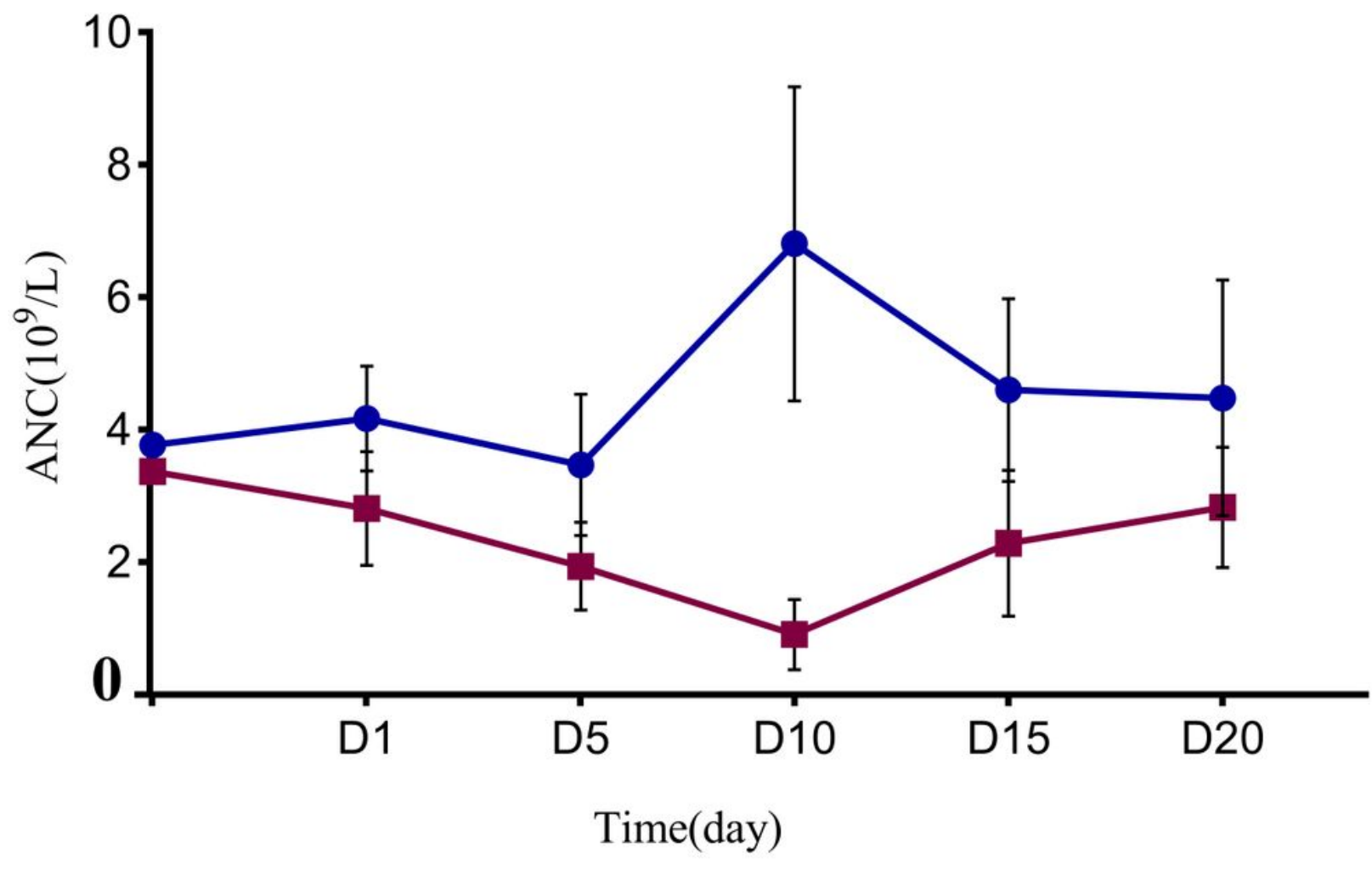

$\rightarrow$ Experimental group $\rightarrow$ Control group

Figure 2

ANC dynamics in the two groups 


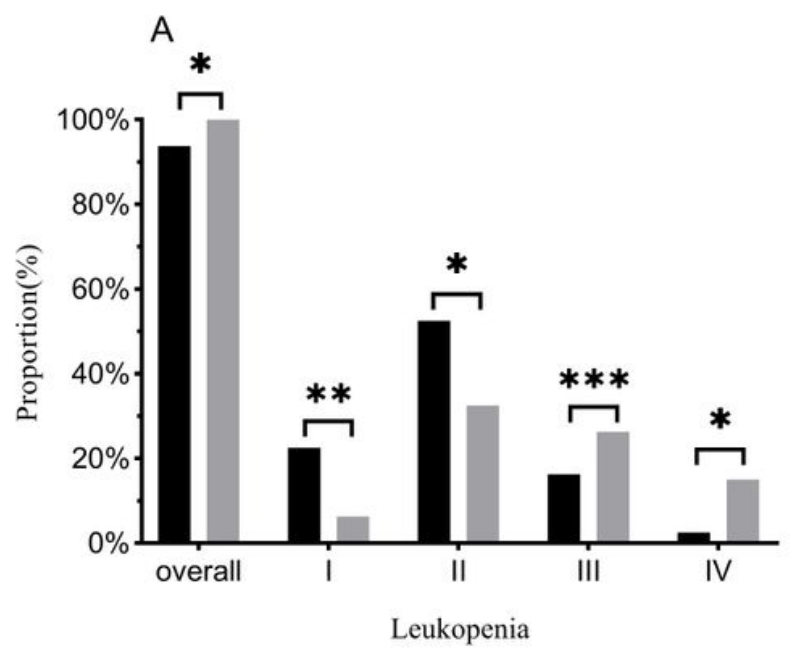

C

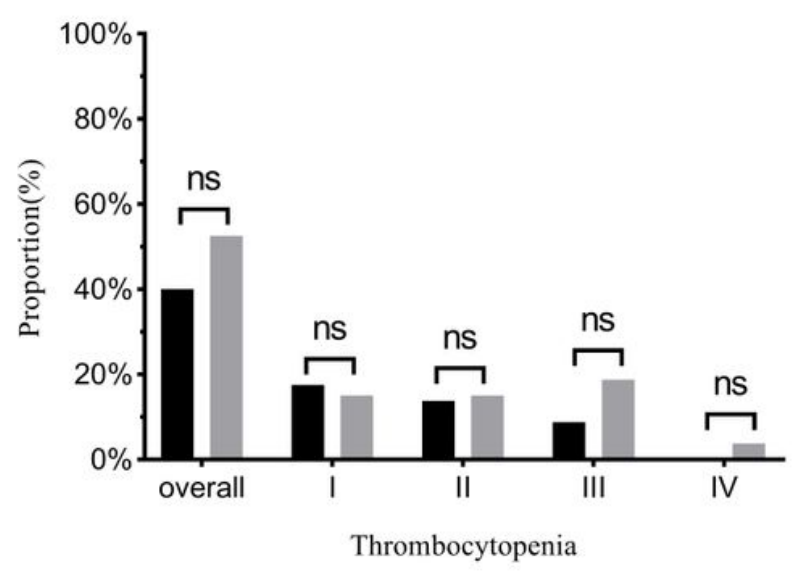

Experimental group( $\mathrm{N}=80)$
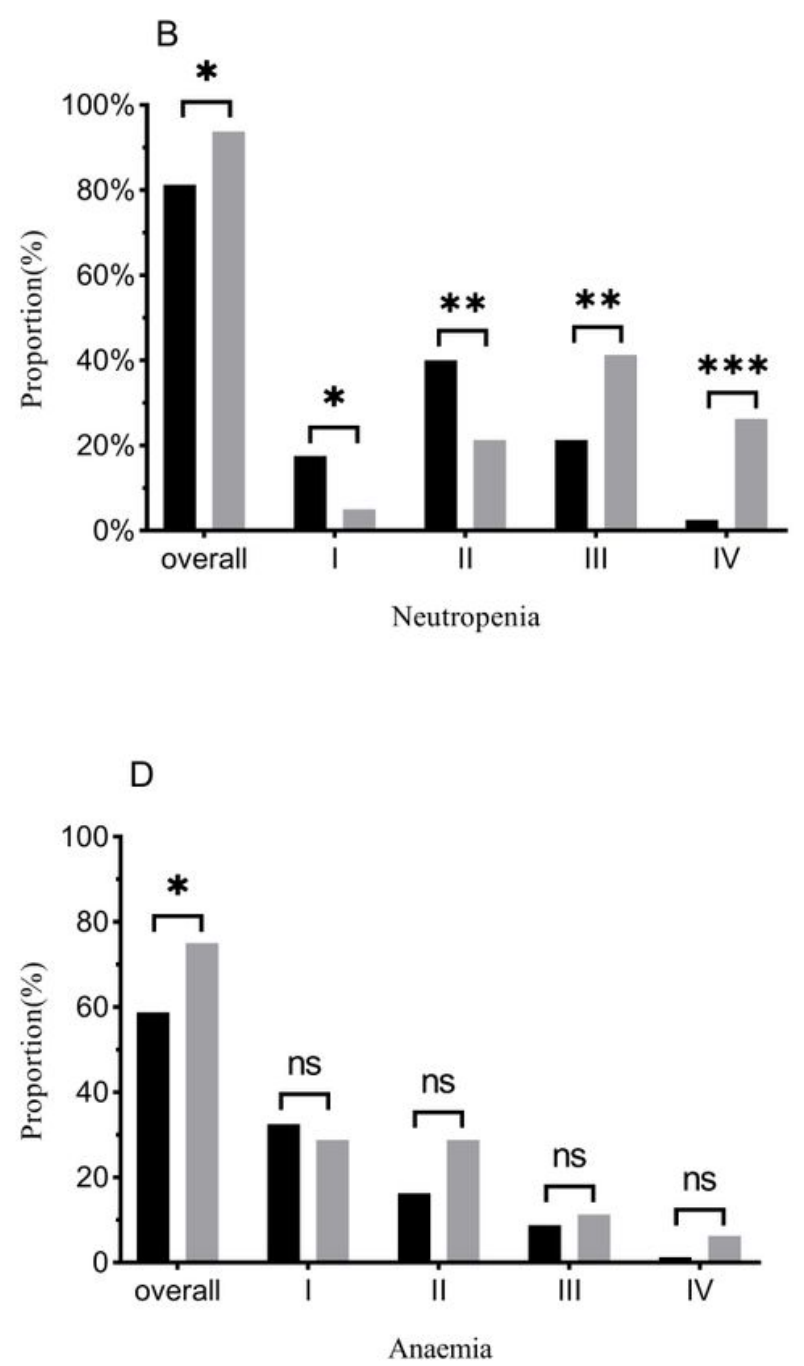

Control group $(\mathrm{N}=80)$

Figure 3

\section{Figure 4}

Radiological reactions, incidence of FN and other adverse reactions

RP: Radiation pneumonia, RE: Radiation esophagitis, FN: Febrile neutropenia

* Factors with statistical significance: ns: no significance; *: $p<0.05 ; * *: p<0.01 ; * \star *: p<0.001$. 


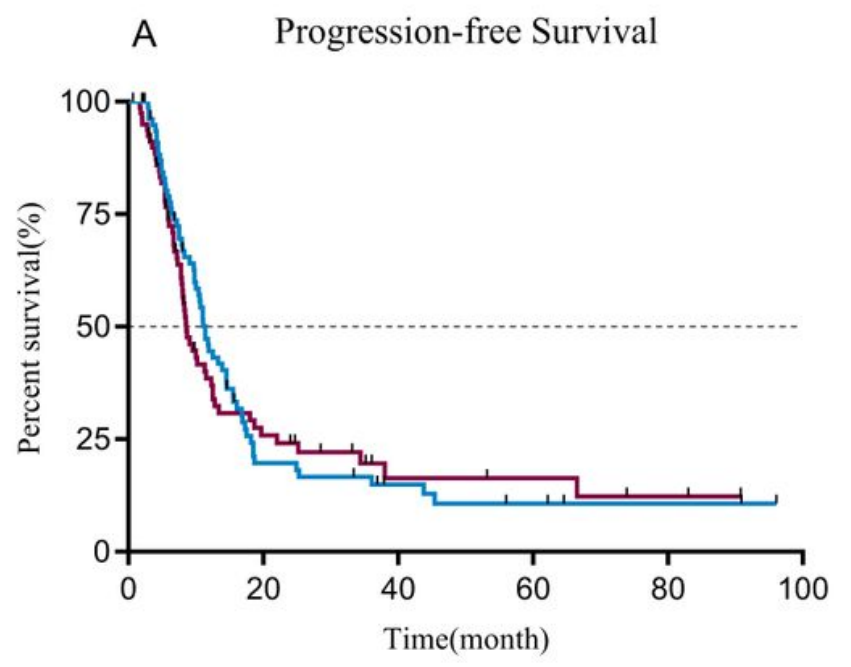

- Experimental group

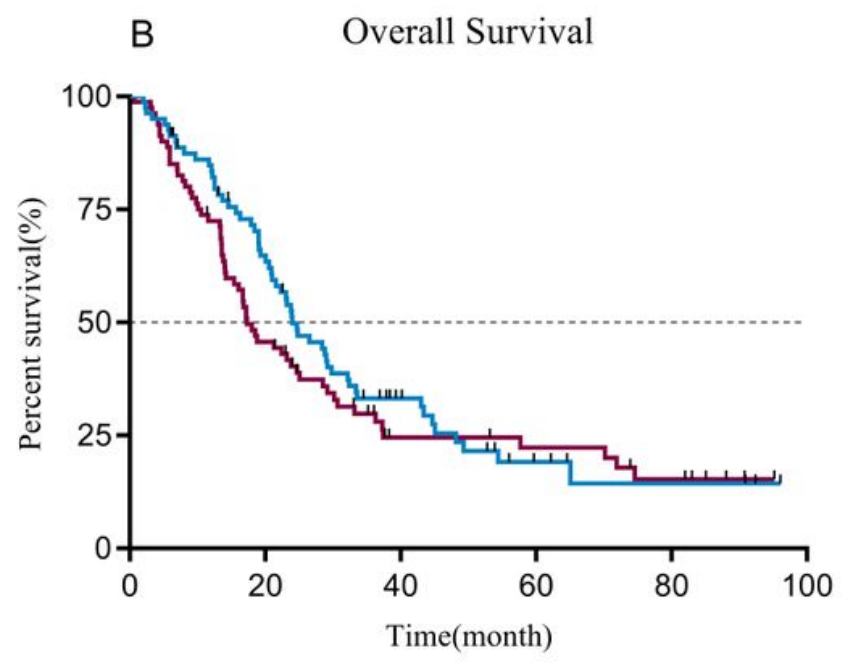

- Control group

Figure 5

Kaplan-Meier curves of PFS (Fig. 5A) and OS (Fig. 5B) of the two groups

\section{Supplementary Files}

This is a list of supplementary files associated with this preprint. Click to download.

- Additionalfile1.doc 